\title{
EXTENDED FULL COMPUTATION-TREE LOGICS FOR PARACONSISTENT MODEL CHECKING
}

\begin{abstract}
It is known that the full computation-tree logic $\mathrm{CTL}^{*}$ is an important base logic for model checking. The bisimulation theorem for CTL* is known to be useful for abstraction in model checking. In this paper, the bisimulation theorems for two paraconsistent four-valued extensions $4 \mathrm{CTL}^{*}$ and $4 \mathrm{LCTL}^{*}$ of $\mathrm{CTL}^{*}$ are shown, and a translation from $4 \mathrm{CTL}^{*}$ into $\mathrm{CTL}^{*}$ is presented. By using $4 \mathrm{CTL}^{*}$ and $4 \mathrm{LCTL}^{*}$, inconsistency-tolerant and spatiotemporal reasoning can be expressed as a model checking framework.
\end{abstract}

Keywords: bisimulation, full computation-tree logic, location operator, paraconsistent model checking.

\section{Introduction}

\subsection{Full computation-tree logic and bisimulation}

Model checking $[10,29,12]$ is an automatic technique for verifying whether a system model $M$, represented as a Kripke structure, satisfies a specification $\alpha$, written as a temporal logic formula. Temporal logics are modal logics for describing the temporal ordering of events. Two possible views ragarding the nature of time induce two types of temporal logics: linear-time temporal logics such as LTL (linear-time temporal logic) [28] and branching-time temporal logics such as CTL (computation-tree logic) [10]. It is known that 
the full computation-tree logic $\mathrm{CTL}^{*}[17,16]$, which is a result of integrating LTL and CTL, is an important base logic for model checking. Since concrete system models tend to be very large, the state explosion problem is arised, and abstraction techniques are needed for reducing a large concrete model to a small abstract one. As presented in $[6,12]$, the following bisimulation theorem for CTL*, which is useful for abstraction in model checking, is well-known: If two Kripke structures $M$ and $M^{\prime}$ are bisimulation equivalent, then for every $\mathrm{CTL}^{*}$ formula $\alpha, M$ satisfies $\alpha$ if and only if $M^{\prime}$ satisfies $\alpha$. This theorem guarantees that we can use an efficient small abstract structure which is bisimular to the given concrete large structure. The logic $\mathrm{CTL}^{*}$ and the bisimulation result addressed above are not sufficient for dealing with inconsistency-tolerant and spatio-temporal reasoning more appropriately. The aim of this paper is thus to extend the framework of $\mathrm{CTL}^{*}$ in order to obtain logical foundations for inconsistency-tolerant and spatio-temporal model checking.

\subsection{Present paper's results}

In this paper, two paraconsistent four-valued extensions $4 \mathrm{CTL}^{*}$ and $4 \mathrm{LCTL}^{*}$ of both CTL* and Nelson's paraconsistent logic N4 with strong negation [1] are introduced, and the bisimulation theorems for $4 \mathrm{CTL}^{*}$ and $4 \mathrm{LCTL}^{*}$ are shown. A translation from $4 \mathrm{CTL}^{*}$ into $\mathrm{CTL}^{*}$ is also presented as an extension of Rautenberg's embedding [31] for Nelson's logics [26]. By using this translation, the existing model checking algorithms for CTL* can be applied to $4 \mathrm{CTL}^{*}$. The logic $4 \mathrm{CTL}^{*}$ (four-valued $\mathrm{CTL}^{*}$ ) is obtained from $\mathrm{CTL}^{*}$ by adding a strong negation operator $\sim$, and the logic $4 \mathrm{LCTL}^{*}$ (locative $\left.4 \mathrm{CTL}^{*}\right)$ is obtained from $4 \mathrm{CTL}^{*}$ by adding a location operator $[l]$. By using these logics, inconsistency-tolerant and spatio-temporal reasoning can be expressed as a model checking framework.

\subsection{Strong negation and paraconsistency}

A remarkable feature of $4 \mathrm{CTL}^{*}$ and $4 \mathrm{LCTL}^{*}$ is that these logics have two kinds of negations: $\sim$ (strong negation) and $\neg$ (usual classical negation in $\left.\mathrm{CTL}^{*}\right)$. The strong negation $\sim$, which was first introduced in [26], has been studied by many researchers, and also applied to various non-classical logics (see e.g. [24, 21, 33, 34] and the references therein). A reason why $\sim$ is added to various logics is that it derives the property of paraconsistency [27]. Roughly speaking, a consequence relation $\models$ is called paraconsistent with 
respect to a negation connective $\sim$ if the following condition holds: $\exists \alpha, \beta$ : formulas, not- $[M, s \models(\alpha \wedge \sim \alpha) \rightarrow \beta]$ where $s$ is a state of a Kripke structure $M$. It is known that logical systems with paraconsistency can deal with inconsistency-tolerant and uncertainty reasoning more appropriately. For example, we do not desire the situation that $(s(x) \wedge \sim s(x)) \rightarrow d(x)$ is satisfied for any symptom $s$ and disease $d$ where $\sim s(x)$ means "a person $x$ does not have a symptom $s$ " and $d(x)$ means "a person $x$ suffers from a disease $d$ ", because symptoms and diseases are vague and uncertain concepts. For more information on paraconsistency, see e.g. [3, 7, 13]. A promising application of $4 \mathrm{CTL}^{*}$ and $4 \mathrm{LCTL}^{*}$ for paraconsistent reasoning may be model checking for requirements elicitation in software engineering. An application of multivalued and paraconsistent model-checking for requirements elicitation was first studied in [14]. In requirements elicitation, different stakeholders often hold different views of how a proposed system should behave, resulting in inconsistencies between their descriptions [14].

\subsection{Verification and refutation}

Another remarkable feature of $4 \mathrm{CTL}^{*}$ and $4 \mathrm{LCTL}^{*}$ is that these logics have two kinds of consequence relations $\models^{+}$(verification) and $\models^{-}$(refutation), which are a new device for model checking. By using these consequence relations, the notion of the 'verification' and the 'refutation or falsification' can simultaneously be represented. We believe that a model-cheker based on such consequence relations can appropriately combine over- and underapproximating abstractions. In software model-checking, typical modelcheckers are used for refutation as well as verification, because of their high bug-finding abilities. A software model-checker Yasm [19, 20] which is based on Belnap's 4-valued logic is the first approach to combine verification and refutation based on the abstraction technique CEGAR [11]. Since Belnap's 4 -valued logic is regarded as a sublogic of Nelson's logic N4 [1], 4CTL* and 4LCTL* may obtain logical justification of such combining approaches.

\subsection{Location operator}

A peculiarity of the locative version $4 \mathrm{LCTL}^{*}$ is that it has the location operator $[l]$ which indicates the location of propositions, e.g. a formula $[l] \alpha$ means "proposition $\alpha$ holds at location $l$." This operator is the same setting as in [22, 23], which is regarded as a refinement of the original proposal of Kobayashi et al. [25]. In [25], such a location operator was introduced 
using a structural congruence relation in formalizing a distributed concurrent linear logic programming language. In [22, 23], the framework of this original operator was improved in order to obtain pure logical foundations without any structural congruence relation. Assuming a space domain Loc and the operator $[l]$ with $l \in$ Loc, a consequence relation $(s, l) \models^{+} \alpha$ of $4 \mathrm{LCTL}^{*}$ can be interpreted as "proposition $\alpha$ holds at time (or state) $s$ and location $l$ ". Various kinds of spatio-temporal situations can thus be expressed using 4LCTL*. For example, a linevenss property: 'If we input the login-password of the host computer Comp3 at one of the mobile computers Comp1 and Comp2, then we will eventually be able to login Comp3' can directly be expressed as

$$
\mathrm{G}([\text { comp1] password } \vee[\text { comp2] password } \rightarrow \mathrm{F}[\text { comp3]login })
$$

where the space domain Loc is $\{c o m p 1, \operatorname{comp} 2, \operatorname{comp} 3\}$, G represents 'anytime in the future', and F represents 'eventually in the future. ${ }^{1}$

\subsection{Paraconsistency-centric approaches}

It is remarked that foundations and applications of multi-valued model checking have been studied by many researchers (see e.g. $[5,8,9,14]$ and the references therein). The present paper's result is also an example of such studies. Paraconsistency-centric approaches to multi-valued model checking were studied in $[14,15]$. In [14], multi-valued belief exploration logic framework for merging and reasoning about inconsistent viewpoints were presented. In [15], an automated tool for paraconsistent reasoning were introduced using a multi-valued model checking framework. Both frameworks were based on the multi-valued computation-tree logic $\chi \mathrm{CTL}$ with the algebraic structures called quasi-Boolean logics. Kripke structures for these frameworks were based on a multi-valued transition relation and a multivalued valuation (labeling) function. The multi-valued valuation function was a very general setting because it can express $n$-valued truth values for any natural number $n$. The present paper's framework is regarded as a special case of these multi-valued frameworks, since the two valuation functions presented in this paper, which are inductively extended to $\models^{-}$and $\models^{+}$, can be transformed into a four-valued valuation function.

\footnotetext{
${ }^{1}$ Strictly speaking, in a CTL* based framework, G and F must be replaced respectively by $A G$ and $A F$ where $A$ is the universal path quantifier.
} 


\subsection{Organization of this paper}

The contents of this paper are then summarized as follows. In Section 2, the logic $4 \mathrm{CTL}^{*}$ is introduced as a Kripke structure with $\models^{+}$and $\models^{-}$, and the bisimulation theorem for $4 \mathrm{CTL}^{*}$ is shown using the method presented in [12]. In Section 3, a translation from $4 \mathrm{CTL}^{*}$ into $\mathrm{CTL}^{*}$, which is an extension of Rautenberg's embedding, is presented using an alternative (intermediate) formulation of $4 \mathrm{CTL}^{*}$ with a single consequence relation $\models$. In Section 4, the logic $4 \mathrm{LCTL}^{*}$ is introduced as a locative Kripke structure with a space domain Loc, and the bisimulation theorem for $4 \mathrm{LCTL}^{*}$ is shown. In Section 5, some illustrative examples such as bipolar preference modeling are presented based on $4 \mathrm{CTL}^{*}$ and $4 \mathrm{LCTL}^{*}$. In Section 6 , this paper is concluded with some remarks.

\section{2. $4 \mathrm{CTL}^{*}$ and bisimulation}

\section{1. $4 \mathrm{CTL}^{*}$}

An expression ATOM means the set of atomic formulas. In the following explanation, we will follow [12]. Formulas of $4 \mathrm{CTL}^{*}$ are defined by combining two types of formulas: state formulas and path formulas.

Definition 2.1. State formulas are obtained by the following rules.

1. If $p \in \mathrm{ATOM}$, then $p$ is a state formula.

2. If $\alpha_{1}$ and $\alpha_{2}$ are state formulas, then $\sim \alpha_{1}, \neg \alpha_{1}, \alpha_{1} \wedge \alpha_{2}, \alpha_{1} \vee \alpha_{2}$ and $\alpha_{1} \rightarrow \alpha_{2}$ are state formulas.

3. If $\alpha$ is a path formula, then $\mathrm{E} \alpha$ and $\mathrm{A} \alpha$ are state formulas.

Path formulas are obtained by the following rules.

1. If $\beta$ is a state formula, then $\operatorname{path}(\beta)$ is a path formula where path is an auxiliary function from the set of state formulas to the set of path formulas. $^{2}$

2. If $\beta_{1}$ and $\beta_{2}$ are path formulas, then $\sim \beta_{1}, \neg \beta_{1}, \beta_{1} \wedge \beta_{2}, \beta_{1} \vee \beta_{2}, \beta_{1} \rightarrow \beta_{2}$, $\mathrm{X} \beta_{1}, \mathrm{~F} \beta_{1}, \mathrm{G} \beta_{1}, \beta_{1} \mathrm{U} \beta_{2}$ and $\beta_{1} \mathrm{R} \beta_{2}$ are path formulas.

State formulas and path formulas are $4 \mathrm{CTL}^{*}$ formulas.

\footnotetext{
${ }^{2}$ Since the function 'path' is usually omitted [12], this rule is interpreted as follows: A state formula is a path formula.
} 
In this definition, E ("some computation path") and A ("all computation paths") are called the "path quantifiers", and X ("next"), F ("eventually"), G ("always"), U ("until") and R ("release") are called the "temporal operators".

In the following, the logic $4 \mathrm{CTL}^{*}$ is defined as a Kripke structure with two consequence relations $\models^{+}$and $\models^{-}$.

Definition 2.2. A Kripke structure is a structure $\left\langle S, S_{0}, R, L^{+}, L^{-}\right\rangle$such that

$S$ is the set of states,

$S_{0}$ is a set of initial states and $S_{0} \subseteq S$,

$R$ is a binary relation on $S$ which satisfies the condition: $\forall s \in$ $S \exists s^{\prime} \in S\left[\left(s, s^{\prime}\right) \in R\right]$, and

$L^{+}$and $L^{-}$are functions from $S$ to the power set of AT $\subseteq$ ATOM.

Definition 2.3. A path in a Kripke structure is an infinite sequence of states, $\pi=s_{0}, s_{1}, s_{2}, \ldots$ such that $\forall i \geqslant 0\left[\left(s_{i}, s_{i+1}\right) \in R\right]$. An expression $\pi^{i}$ means the suffix of $\pi$ starting at $s_{i}$.

DEFInition 2.4. Let AT be a nonempty subset of ATOM. Let $\alpha_{1}$ and $\alpha_{2}$ be state formulas and $\beta_{1}$ and $\beta_{2}$ be path formulas. Consequence relations $\models^{+}$ and $\models^{-}$on a Kripke structure $M=\left\langle S, S_{0}, R, L^{+}, L^{-}\right\rangle$are defined inductively as follows ( $\pi$ represents a path constructed from $S$, and $s$ represents a state in $S$ ):

1. $M, s \models^{+} p$ iff $p \in L^{+}(s)$ for $p \in \mathrm{AT}$,

2. $M, s \models^{-} p$ iff $p \in L^{-}(s)$ for $p \in \mathrm{AT}$,

3. $M, s \models^{+} \sim \alpha_{1}$ iff $M, s \models^{-} \alpha_{1}$,

4. $M, s \models^{+} \neg \alpha_{1}$ iff not-[M,s $\left.\models^{+} \alpha_{1}\right]$,

5. $M, s \models^{+} \alpha_{1} \wedge \alpha_{2}$ iff $M, s \models^{+} \alpha_{1}$ and $M, s \models^{+} \alpha_{2}$,

6. $M, s \models^{+} \alpha_{1} \vee \alpha_{2}$ iff $M, s \models^{+} \alpha_{1}$ or $M, s \models^{+} \alpha_{2}$,

7. $M, s \models^{+} \alpha_{1} \rightarrow \alpha_{2}$ iff $M, s \models^{+} \alpha_{1}$ implies $M, s \models{ }^{+} \alpha_{2}$,

8. $M, s \models^{+} \mathrm{E} \beta_{1}$ iff there exists a path $\pi$ from $s$ such that $M, \pi \models^{+} \beta_{1}$,

9. $M, s \models^{+} \mathrm{A} \beta_{1}$ iff for every path $\pi$ starting from $s, M, \pi \models^{+} \beta_{1}$,

10. $M, \pi \models^{+} \operatorname{path}\left(\alpha_{1}\right)$ iff $s$ is the first state of $\pi$ and $M, s \models^{+} \alpha_{1}$,

11. $M, \pi \models^{+} \sim \beta_{1}$ iff $M, \pi \models^{-} \beta_{1}$,

12. $M, \pi \models^{+} \neg \beta_{1}$ iff not-[M, $\left.\pi \models^{+} \beta_{1}\right]$, 
13. $M, \pi \models^{+} \beta_{1} \wedge \beta_{2}$ iff $M, \pi \models^{+} \beta_{1}$ and $M, \pi \models^{+} \beta_{2}$,

14. $M, \pi \models^{+} \beta_{1} \vee \beta_{2}$ iff $M, \pi \models^{+} \beta_{1}$ or $M, \pi \models^{+} \beta_{2}$,

15. $M, \pi \models^{+} \beta_{1} \rightarrow \beta_{2}$ iff $M, \pi \models^{+} \beta_{1}$ implies $M, \pi \models^{+} \beta_{2}$,

16. $M, \pi \models^{+} \mathrm{X} \beta_{1}$ iff $M, \pi^{1} \models^{+} \beta_{1}$,

17. $M, \pi \models^{+} \mathrm{F} \beta_{1}$ iff $\exists k \geqslant 0\left[M, \pi^{k} \models^{+} \beta_{1}\right]$,

18. $M, \pi \models^{+} \mathrm{G} \beta_{1}$ iff $\forall k \geqslant 0\left[M, \pi^{k} \models^{+} \beta_{1}\right]$,

19. $M, \pi \models^{+} \beta_{1} \mathrm{U} \beta_{2}$ iff $\exists k \geqslant 0\left[\left(M, \pi^{k} \models^{+} \beta_{2}\right)\right.$ and $\forall j(0 \leqslant j<k$ implies $\left.\left.M, \pi^{j} \models^{+} \beta_{1}\right)\right]$

20. $M, \pi \models^{+} \beta_{1} \mathrm{R} \beta_{2}$ iff $\forall j \geqslant 0\left[\forall i<j\right.$ not- $\left[M, \pi^{i} \models^{+} \beta_{1}\right]$ implies $M, \pi^{j} \models{ }^{+} \beta_{2}$ ]

21. $M, s \models^{-} \sim \alpha_{1}$ iff $M, s \models^{+} \alpha_{1}$,

22. $M, s \models-\neg \alpha_{1}$ iff not-[M,s $\left[\models^{-} \alpha_{1}\right]$,

23. $M, s \models^{-} \alpha_{1} \wedge \alpha_{2}$ iff $M, s \models^{-} \alpha_{1}$ or $M, s \models^{-} \alpha_{2}$,

24. $M, s \models^{-} \alpha_{1} \vee \alpha_{2}$ iff $M, s \models^{-} \alpha_{1}$ and $M, s \models^{-} \alpha_{2}$,

25. $M, s \models^{-} \alpha_{1} \rightarrow \alpha_{2}$ iff $M, s \models^{+} \alpha_{1}$ and $M, s \models^{-} \alpha_{2}$,

26. $M, s \models^{-} \mathrm{E} \beta_{1}$ iff for every path $\pi$ starting from $s, M, \pi \models^{-} \beta_{1}$,

27. $M, s \models^{-} \mathrm{A} \beta_{1}$ iff there exists a path $\pi$ from $s$ such that $M, \pi \models^{-} \beta_{1}$,

28. $M, \pi \models^{-} \operatorname{path}\left(\alpha_{1}\right)$ iff $s$ is the first state of $\pi$ and $M, s \models^{-} \alpha_{1}$,

29. $M, \pi \models^{-} \sim \beta_{1}$ iff $M, \pi \models^{+} \beta_{1}$,

30. $M, \pi \models^{-} \neg \beta_{1}$ iff not- $\left[M, \pi \models^{-} \beta_{1}\right]$,

31. $M, \pi \models^{-} \beta_{1} \wedge \beta_{2}$ iff $M, \pi \models^{-} \beta_{1}$ or $M, \pi \models^{-} \beta_{2}$,

32. $M, \pi \models^{-} \beta_{1} \vee \beta_{2}$ iff $M, \pi \models^{-} \beta_{1}$ and $M, \pi \models^{-} \beta_{2}$,

33. $M, \pi \models^{-} \beta_{1} \rightarrow \beta_{2}$ iff $M, \pi \models^{+} \beta_{1}$ and $M, \pi \models^{-} \beta_{2}$,

34. $M, \pi \models^{-} \mathrm{X} \beta_{1}$ iff $M, \pi^{1} \models^{-} \beta_{1}$,

35. $M, \pi \models^{-} \mathrm{F} \beta_{1}$ iff $\forall k \geqslant 0\left[M, \pi^{k} \models^{-} \beta_{1}\right]$,

36. $M, \pi \models^{-} \mathrm{G} \beta_{1}$ iff $\exists k \geqslant 0\left[M, \pi^{k} \models^{-} \beta_{1}\right]$,

37. $M, \pi \models^{-} \beta_{1} \mathrm{U} \beta_{2}$ iff $\forall j \geqslant 0\left[\forall i<j\right.$ not- $\left[M, \pi^{i} \models^{-} \beta_{1}\right]$ implies $\left.M, \pi^{j}=-\beta_{2}\right]$,

38. $M, \pi \models^{-} \beta_{1} \mathrm{R} \beta_{2}$ iff $\exists k \geqslant 0\left[\left(M, \pi^{k} \models^{-} \beta_{2}\right)\right.$ and $\forall j(0 \leqslant j<k$ implies $\left.\left.M, \pi^{j} \models^{-} \beta_{1}\right)\right]$.

The notation $\models^{*}$ is used for $\models^{+}$or $\models^{-} . \quad M \models^{*} \alpha$ is defined by $\forall s \in S$ $\left[M, s \models{ }^{*} \alpha\right]$. 
Since $\models^{+}$is the usual consequence relation $\models$ of $\mathrm{CTL}^{*}$ and $\models^{-}$is the De Morgan dual of $\models^{+}, 3$ the intuitive interpretations of $\models^{+}$and $\models^{-}$are "verification" and "refutation or falsification", respectively. Roughly speaking, to introduce $\models^{-}$is thus allow to obtain the following axiom schemes w.r.t. $\sim$, where $\alpha \leftrightarrow \beta$ means $(\alpha \rightarrow \beta) \wedge(\beta \rightarrow \alpha)$.

$$
\begin{aligned}
\sim \sim \alpha & \leftrightarrow \alpha, \\
\sim \neg \alpha & \leftrightarrow \neg \sim \alpha, \\
\sim(\alpha \wedge \beta) & \leftrightarrow \sim \alpha \vee \sim \beta, \\
\sim(\alpha \vee \beta) & \leftrightarrow \sim \alpha \wedge \sim \beta, \\
\sim(\alpha \rightarrow \beta) & \leftrightarrow \alpha \wedge \sim \beta, \\
\sim \mathrm{X} \alpha & \leftrightarrow \mathrm{X} \sim \alpha, \\
\sim \mathrm{F} \alpha & \leftrightarrow \mathrm{G} \sim \alpha, \\
\sim \mathrm{G} \alpha & \leftrightarrow \mathrm{F} \sim \alpha, \\
\sim \mathrm{E} \alpha & \leftrightarrow \mathrm{A} \sim \alpha, \\
\sim \mathrm{A} \alpha & \leftrightarrow \mathrm{E} \sim \alpha, \\
\sim \mathrm{path}(\alpha) & \leftrightarrow \mathrm{path}(\sim \alpha), \\
\sim\left(\alpha_{1} \mathrm{U} \alpha_{2}\right) & \leftrightarrow\left(\sim \alpha_{1}\right) \mathrm{R}\left(\sim \alpha_{2}\right), \\
\sim\left(\alpha_{1} \mathrm{R} \alpha_{2}\right) & \leftrightarrow\left(\sim \alpha_{1}\right) \mathrm{U}\left(\sim \alpha_{2}\right) .
\end{aligned}
$$

For each state $s$ and each formula $\alpha$, we can take one of the following four cases: (1) $\alpha$ is verified at $s$ (i.e. $M, s \models^{+} \alpha$ ), (2) $\alpha$ is falsified at $s$ (i.e. $M, s \models^{-} \alpha$ ), (3) $\alpha$ is both verified and falsified at $s$, and (4) $\alpha$ is neither verified nor falsified at $s$. Thus, $4 \mathrm{CTL}^{*}$ is regarded as a four-valued logic.

Assume a Kripke structure $M=\left\langle S, S_{0}, R, L^{+}, L^{-}\right\rangle$such that $p \in L^{+}(s)$, $p \in L^{-}(s)$ and $q \notin L^{+}(s)$ for any distinct atomic formulas $p$ and $q$. Then, $M, s \models^{+}(p \wedge \sim p) \rightarrow q$ does not hold, and hence $\models^{+}$in $4 \mathrm{CTL}^{*}$ is paraconsistent with respect to $\sim$.

\subsection{Bisimulation}

Definition 2.5. Let $M=\left\langle S, S_{0}, R, L^{+}, L^{-}\right\rangle$and $M^{\prime}=\left\langle S^{\prime}, S_{0}^{\prime}, R^{\prime}, L^{\prime+}, L^{\prime-}\right\rangle$ be Kripke structures with the same nonempty set AT $\subseteq$ ATOM.

A relation $B \subseteq S \times S^{\prime}$ is a bisimulation relation between $M$ and $M^{\prime}$ if and only if for all $s$ and $s^{\prime}$, if $B\left(s, s^{\prime}\right)$ then the following conditions hold.

1. $L^{+}(s)=L^{\prime+}\left(s^{\prime}\right)$,

\footnotetext{
${ }^{3}$ Except the cases for $\mathrm{X}$, path and $\neg$. These cases are adapted to be the self-dual.
} 
2. $L^{-}(s)=L^{\prime-}\left(s^{\prime}\right)$,

3. $\forall s_{1}\left[R\left(s, s_{1}\right)\right.$ implies $\exists s_{1}^{\prime}\left[R^{\prime}\left(s^{\prime}, s_{1}^{\prime}\right)\right.$ and $\left.\left.B\left(s_{1}, s_{1}^{\prime}\right)\right]\right]$,

4. $\forall s_{1}^{\prime}\left[R^{\prime}\left(s^{\prime}, s_{1}^{\prime}\right)\right.$ implies $\exists s_{1}\left[R\left(s, s_{1}\right)\right.$ and $\left.\left.B\left(s_{1}, s_{1}^{\prime}\right)\right]\right]$.

The structures $M$ and $M^{\prime}$ are bisimulation equivalent if there exists a bisimulation relation $B$ such that (1) $\forall s_{0} \in S_{0} \exists s_{0}^{\prime} \in S_{0}^{\prime}\left[B\left(s_{0}, s_{0}^{\prime}\right)\right]$ and (2) $\forall s_{0}^{\prime} \in S_{0}^{\prime} \exists s_{0} \in S_{0}\left[B\left(s_{0}, s_{0}^{\prime}\right)\right]$.

Definition 2.6. Two paths $\pi=s_{0}, s_{1}, s_{2}, \ldots$ in a Kripke structure $M$ and $\pi^{\prime}=s_{0}^{\prime}, s_{1}^{\prime}, s_{2}^{\prime}, \ldots$ in a Kripke structure $M^{\prime}$ are called corresponding paths if $\forall i \geqslant 0\left[B\left(s_{i}, s_{i}^{\prime}\right)\right]$.

Lemma 2.7. Let $s$ and $s^{\prime}$ be two states such that $B\left(s, s^{\prime}\right)$. Then for every path starting from $s$ there is a corresponding path starting from $s^{\prime}$, and for every path starting from $s^{\prime}$ there is a corresponding path starting from $s$.

Proof. See [12].

Lemma 2.8. Let $M$ and $M^{\prime}$ be Kripke structures with the same nonempty set $\mathrm{AT} \subseteq \mathrm{ATOM}$, and $B$ be a bisimulation relation between $M$ and $M^{\prime}$. Let $\alpha$ be a $4 \mathrm{CTL}^{*}$ formula. Assume that $B\left(s, s^{\prime}\right)$ and that $\pi$ in $M$ and $\pi^{\prime}$ in $M^{\prime}$ are corresponding paths.

If $\alpha$ is a state formula, then

(1) $M, s \models^{+} \alpha \quad$ iff $\quad M^{\prime}, s^{\prime} \models^{+} \alpha$,

(2) $M, s \models{ }^{-} \alpha \quad$ iff $\quad M^{\prime}, s^{\prime} \models^{-} \alpha$,

and if $\alpha$ is a path formula, then

(1) $M, \pi \models^{+} \alpha \quad$ iff $\quad M^{\prime}, \pi^{\prime} \models^{+} \alpha$,

(2) $M, \pi \models^{-} \alpha \quad$ iff $\quad M^{\prime}, \pi^{\prime} \models^{-} \alpha$.

Proof. This lemma is proved by (simultaneous) induction on the complexity of $\alpha$. In the following, the Kripke structures $M$ and $M^{\prime}$ are omitted in the expressions, since the structures are clear from the context.

Base step: $\alpha \equiv p$ for $p \in$ AT. By the assumption $B\left(s, s^{\prime}\right)$, we have $L^{+}(s)=L^{\prime+}\left(s^{\prime}\right)$ and $L^{-}(s)=L^{\prime-}\left(s^{\prime}\right)$, and hence obtain (1) $s \models^{+} p$ iff $s^{\prime} \models^{+}$ $p$ and (2) $s \models^{-} p$ iff $s^{\prime} \models^{-} p$.

Induction step: We show only some cases.

(Case $\alpha \equiv \alpha_{1} \vee \alpha_{2}$ is a state formula): (1) $s \models^{+} \alpha_{1} \vee \alpha_{2}$ iff $s \models^{+} \alpha_{1}$ or $s \models{ }^{+} \alpha_{2}$ iff $s^{\prime} \models^{+} \alpha_{1}$ or $s^{\prime} \models^{+} \alpha_{2}$ (induction hypothesis) iff $s^{\prime} \models^{+} \alpha_{1} \vee \alpha_{2}$. 
(2) $s \models^{-} \alpha_{1} \vee \alpha_{2} \quad$ iff $s \models^{-} \alpha_{1}$ and $s \models^{-} \alpha_{2} \quad$ iff $\quad s^{\prime} \models^{-} \alpha_{1}$ and $s^{\prime} \models^{-} \alpha_{2}$ (induction hypothesis) iff $s^{\prime} \models^{-} \alpha_{1} \vee \alpha_{2}$.

(Case $\alpha \equiv \alpha_{1} \vee \alpha_{2}$ is a path formula): Similar to the above case.

(Case $\alpha \equiv \sim \alpha_{1}$ is a state formula) $)^{4}:$ (1) $s \models^{+} \sim \alpha_{1}$ iff $s \models^{-} \alpha_{1}$ iff $s^{\prime} \models^{-} \alpha_{1}$ (induction hypothesis) iff $s^{\prime} \models^{+} \sim \alpha_{1}$. (2) $s \models^{-} \sim \alpha_{1}$ iff $s \models^{+} \alpha_{1}$ iff $s^{\prime} \models^{+} \alpha_{1}$ (induction hypothesis) iff $s^{\prime} \models^{-} \sim \alpha_{1}$.

(Case $\alpha \equiv \sim \alpha_{1}$ is a path formula): Similar to the above case.

(Case $\alpha \equiv \mathrm{A} \alpha_{1}$, a state formula): We only show: $s \models^{*} \alpha$ implies $s^{\prime} \models^{*} \alpha$. The converse direction can be proved in a similar way, and hence omitted. We only consider the case for $*=-$. Suppose $s \models^{-} \mathrm{A} \alpha_{1}$. Then we have that $\exists \pi_{1}$ : path starting form $s\left[\pi_{1} \models^{-} \alpha_{1}\right]$. By Lemma 2.7, we have that $(*)$ : there exists a corresponding path $\pi_{1}^{\prime}$ starting from $s^{\prime}$, i.e. $\pi_{1}$ and $\pi_{1}^{\prime}$ are corresponding paths. By $(*)$ and the induction hypothesis, we obtain $(* *)$ : $\pi_{1} \models^{-} \alpha_{1}$ iff $\pi_{1}^{\prime} \models^{-} \alpha_{1}$. By $\left(^{*}\right)$ and $\left({ }^{*}\right)$, we obtain that $\exists \pi_{1}^{\prime}$ : path starting from $s^{\prime}\left[\pi_{1}^{\prime} \models^{-} \alpha_{1}\right]$. Therefore $s^{\prime} \models^{-} \mathrm{A} \alpha_{1}$.

(Case $\alpha \equiv \operatorname{path}\left(\alpha_{1}\right)$, a path formula): Let $s_{0}$ and $s_{0}^{\prime}$ be the first states of $\pi$ and $\pi^{\prime}$, respectively. $\pi \models^{*}$ path $\left(\alpha_{1}\right)$ iff $s_{0} \models^{*} \alpha_{1}$ iff $s_{0}^{\prime} \models^{*} \alpha_{1}$ (induction hypothesis) iff $\pi^{\prime} \models^{*} \operatorname{path}\left(\alpha_{1}\right)$.

(Case $\alpha \equiv \mathrm{X} \alpha_{1}$, a path formula): We only show: $\pi \models^{*} \alpha_{1}$ implies $\pi^{\prime} \models^{*} \alpha_{1}$. The converse direction can be shown in a similar way. Suppose $\pi \models{ }^{*} \mathrm{X} \alpha_{1}$. Then we have $\pi^{1} \models \alpha_{1}$. Since $\pi$ and $\pi^{\prime}$ are corresponding paths by the assumption, so are $\pi^{1}$ and $\pi^{\prime 1}$. Thus we obtain $\pi^{\prime 1} \models^{*} \alpha_{1}$ by the induction hypothesis, and hence $\pi^{\prime} \models{ }^{*} \mathrm{X} \alpha_{1}$.

(Case $\alpha \equiv \alpha_{1} \operatorname{R} \alpha_{2}$, a path formula): We only show: $\pi \models^{*} \alpha$ implies $\pi^{\prime} \models^{*} \alpha$. The converse direction can be shown in a similar way. We only consider the case for $*=-$. Suppose $\pi \models^{-} \alpha_{1} \operatorname{R} \alpha_{2}$. Then we have $\exists k \geqslant 0$ $\left[\left(\pi^{k} \models^{-} \alpha_{2}\right)\right.$ and $\forall j\left(0 \leqslant j<k\right.$ implies $\left.\left.\pi^{j} \models^{-} \alpha_{1}\right)\right]$. Since by the assumption, $\pi$ and $\pi^{\prime}$ are corresponding paths, $\pi^{j}$ and $\pi^{\prime j}$ are corresponding paths for any $j$. Thus, by the induction hypothesis, we obtain $\pi^{\prime k} \models^{-} \alpha_{2}$ and $\forall j(0 \leqslant j<k$ implies $\pi^{\prime j} \models^{-} \alpha_{1}$ ). Therefore $\pi^{\prime} \models^{-} \alpha_{1} R \alpha_{2}$.

The next theorem is a consequence of the preceding lemma.

Theorem 2.9. Let $M$ and $M^{\prime}$ be Kripke structures and $B$ be bisimulation relation between $M$ and $M^{\prime}$. If $B\left(s, s^{\prime}\right)$, then for every $4 \mathrm{CTL}^{*}$ formula $\alpha$,

(1) $M, s \models^{+} \alpha \quad$ iff $\quad M^{\prime}, s^{\prime} \models^{+} \alpha$,

(2) $M, s \models-\alpha \quad$ iff $\quad M^{\prime}, s^{\prime} \models^{-} \alpha$.

\footnotetext{
${ }^{4}$ In this case, we use the simultaneous induction hypothesis with respect to both $\models^{+}$ and $\models^{-}$.
} 
We then obtain the following theorem.

Theorem 2.10. Suppose that Kripke structures $M$ and $M^{\prime}$ are bisimulation equivalent. For every $4 \mathrm{CTL}^{*}$ formula $\alpha$,

(1) $M \models^{+} \alpha$ iff $M^{\prime} \models^{+} \alpha$,

(2) $M \models^{-} \alpha$ iff $M^{\prime} \models^{-} \alpha$.

\section{Translation from $4 \mathrm{CTL}^{*}$ into $\mathrm{CTL}^{*}$}

In order to obtain a translation from $4 \mathrm{CTL}^{*}$ into $\mathrm{CTL}^{*}$, an alternative formulation of $4 \mathrm{CTL}^{*}$ is defined as a Kripke structure with a single consequence relation $\models$.

Definition 3.11. Let AT1 and AT2 be nonempty subsets of ATOM and $\mathrm{AT}^{\sim}=\{\sim p \mid p \in \mathrm{AT} 2\}$. A single-consequence Kripke structure is a structure $\left\langle S, S_{0}, R, L\right\rangle$ such that $S, S_{0}$ and $R$ are the same as that in Definition 2.2, and

$L$ is a function from $S$ to the power set of $\mathrm{AT} 1 \cup \mathrm{AT} 2^{\sim}$.

A path in a single-consequence Kripke structure is defined in a similar way as in Definition 2.3.

Definition 3.12. Let $\alpha_{1}$ and $\alpha_{2}$ be state formulas and $\beta_{1}$ and $\beta_{2}$ be path formulas. A consequence relation $\models$ on a single-consequence Kripke structure $M=\left\langle S, S_{0}, R, L\right\rangle$ is defined inductively as follows ( $\pi$ represents a path constructed from $S$, and $s$ represents a state in $S$ ):

1. $M, s \models p$ iff $p \in L(s)$ for $p \in \mathrm{AT} 1$,

2. $M, s \models \neg \alpha_{1}$ iff $\operatorname{not}-\left[M, s \models \alpha_{1}\right]$,

3. $M, s \models \alpha_{1} \wedge \alpha_{2}$ iff $M, s \models \alpha_{1}$ and $M, s \models \alpha_{2}$,

4. $M, s \models \alpha_{1} \vee \alpha_{2}$ iff $M, s \models \alpha_{1}$ or $M, s \models \alpha_{2}$,

5. $M, s \models \alpha_{1} \rightarrow \alpha_{2}$ iff $M, s \models \alpha_{1}$ implies $M, s \models \alpha_{2}$,

6. $M, s \models \mathrm{E} \beta_{1}$ iff there exists a path $\pi$ from $s$ such that $M, \pi \models \beta_{1}$,

7. $M, s \models \mathrm{A} \beta_{1}$ iff for every path $\pi$ starting from $s, M, \pi \models \beta_{1}$,

8. $M, \pi \models \operatorname{path}\left(\alpha_{1}\right)$ iff $s$ is the first state of $\pi$ and $M, s \models \alpha_{1}$,

9. $M, \pi \models \neg \beta_{1}$ iff not- $\left[M, \pi \models \beta_{1}\right]$, 
10. $M, \pi \models \beta_{1} \wedge \beta_{2}$ iff $M, \pi \models \beta_{1}$ and $M, \pi \models \beta_{2}$,

11. $M, \pi \models \beta_{1} \vee \beta_{2}$ iff $M, \pi \models \beta_{1}$ or $M, \pi \models \beta_{2}$,

12. $M, \pi \models \beta_{1} \rightarrow \beta_{2}$ iff $M, \pi \models \beta_{1}$ implies $M, \pi \models \beta_{2}$,

13. $M, \pi \models \mathrm{X} \beta_{1}$ iff $M, \pi^{1} \models \beta_{1}$,

14. $M, \pi \models \mathrm{F} \beta_{1}$ iff $\exists k \geqslant 0\left[M, \pi^{k} \models \beta_{1}\right]$,

15. $M, \pi \models \mathrm{G} \beta_{1}$ iff $\forall k \geqslant 0\left[M, \pi^{k} \models \beta_{1}\right]$,

16. $M, \pi \models \beta_{1} \mathrm{U} \beta_{2}$ iff $\exists k \geqslant 0\left[\left(M, \pi^{k} \models \beta_{2}\right)\right.$ and $\forall j(0 \leqslant j<k$ implies $\left.\left.M, \pi^{j} \models \beta_{1}\right)\right]$,

17. $M, \pi \models \beta_{1} \mathrm{R} \beta_{2}$ iff $\forall j \geqslant 0\left[\forall i<j\right.$ not- $\left[M, \pi^{i} \models \beta_{1}\right]$ implies $\left.M, \pi^{j} \models \beta_{2}\right]$,

18. $M, s \models \sim p$ iff $\sim p \in L(s)$ for $\sim p \in \mathrm{AT}^{\sim}$,

19. $M, s \models \sim \sim \alpha_{1}$ iff $M, s \models \alpha_{1}$,

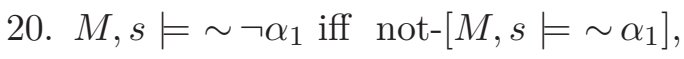

21. $M, s \models \sim\left(\alpha_{1} \wedge \alpha_{2}\right)$ iff $M, s \models \sim \alpha_{1}$ or $M, s \models \sim \alpha_{2}$,

22. $M, s \models \sim\left(\alpha_{1} \vee \alpha_{2}\right)$ iff $M, s \models \sim \alpha_{1}$ and $M, s \models \sim \alpha_{2}$,

23. $M, s \models \sim\left(\alpha_{1} \rightarrow \alpha_{2}\right)$ iff $M, s \models \alpha_{1}$ and $M, s \models \sim \alpha_{2}$,

24. $M, s \models \sim \mathrm{E} \beta_{1}$ iff for every path $\pi$ starting from $s, M, \pi \models \sim \beta_{1}$,

25. $M, s \models \sim \mathrm{A} \beta_{1}$ iff there exists a path $\pi$ from $s$ such that $M, \pi \models \sim \beta_{1}$,

26. $M, \pi \models \sim \operatorname{path}\left(\alpha_{1}\right)$ iff $s$ is the first state of $\pi$ and $M, s \models \sim \alpha_{1}$,

27. $M, \pi \models \sim \sim \beta_{1}$ iff $M, \pi \models \beta_{1}$,

28. $M, \pi \models \sim \neg \beta_{1}$ iff not-[M, $\left.\pi \models \sim \beta_{1}\right]$,

29. $M, \pi \models \sim\left(\beta_{1} \wedge \beta_{2}\right)$ iff $M, \pi \models \sim \beta_{1}$ or $M, \pi \models \sim \beta_{2}$,

30. $M, \pi \models \sim\left(\beta_{1} \vee \beta_{2}\right)$ iff $M, \pi \models \sim \beta_{1}$ and $M, \pi \models \sim \beta_{2}$,

31. $M, \pi \models \sim\left(\beta_{1} \rightarrow \beta_{2}\right)$ iff $M, \pi \models \beta_{1}$ and $M, \pi \models \sim \beta_{2}$,

32. $M, \pi \models \sim \mathrm{X} \beta_{1}$ iff $M, \pi^{1} \models \sim \beta_{1}$,

33. $M, \pi \models \sim \mathrm{F} \beta_{1}$ iff $\forall k \geqslant 0\left[M, \pi^{k} \models \sim \beta_{1}\right]$,

34. $M, \pi \models \sim \mathrm{G} \beta_{1}$ iff $\exists k \geqslant 0\left[M, \pi^{k} \models \sim \beta_{1}\right]$,

35. $M, \pi \models \sim\left(\beta_{1} \mathrm{U} \beta_{2}\right)$ iff $\forall j \geqslant 0\left[\forall i<j\right.$ not- $\left[M, \pi^{i} \models \sim \beta_{1}\right]$ implies $M, \pi^{j} \models$ $\left.\sim \beta_{2}\right]$,

36. $M, \pi \models \sim\left(\beta_{1} \mathrm{R} \beta_{2}\right)$ iff $\exists k \geqslant 0\left[\left(M, \pi^{k} \models \sim \beta_{2}\right)\right.$ and $\forall j(0 \leqslant j<k$ implies $\left.\left.M, \pi^{j} \models \sim \beta_{1}\right)\right]$. 
An equivalence between the single-consequence Kripke structure with $\models$ and the Kripke structure with $\models^{+}$and $\models^{-}$is shown below.

Theorem 3.13. Let AT, AT1 and AT2 be nonempty subset of ATOM and $\mathrm{AT}^{\sim}:=\{\sim p \mid p \in \mathrm{AT} 2\}$. Suppose that $M$ is a Kripke structure $\left\langle S, S_{0}, R, L^{+}, L^{-}\right\rangle$with $L^{+}, L^{-}: S \rightarrow 2^{\mathrm{AT}}, N$ is a single-consequence Kripke structure $\left\langle S, S_{0}, R, L\right\rangle$ with $L: S \rightarrow 2^{\mathrm{AT} 1} \cup \mathrm{AT} 2^{\sim}$, and moreover satisfyning that for any $s \in S,\left[p \in L(s)\right.$ iff $\left.p \in L^{+}(s)\right]$ and $\left[\sim p \in L(s)\right.$ iff $\left.p \in L^{-}(s)\right]$. Let $s$ be a state in $S$ or a path in $M$ and $N$. For any $4 \mathrm{CTL}^{*}$ formula $\alpha$,

(1) $M, s \models^{+} \alpha \quad$ iff $\quad N, s \models \alpha$,

(2) $M, s \models^{-} \alpha \quad$ iff $\quad N, s \models \sim \alpha$.

Proof. This theorem is proved by (simultaneous) induction on the complexity of $\alpha$.

Base step:

(Case $\alpha \equiv p$ for $p \in \mathrm{ATOM}):(1): M, s \models^{+} p$ iff $p \in L^{+}(s)$ iff $p \in L(s)$ iff $N, s \models p$. (2): $M, s \models^{-} p$ iff $p \in L^{-}(s)$ iff $\sim p \in L(s)$ iff $N, s \models \sim p$.

(Case $\alpha \equiv \sim p$ for $p \in \mathrm{ATOM}$ ): (1): $M, s \models^{+} \sim p$ iff $M, s \models^{-} p$ iff $p \in L^{-}(s)$ iff $\sim p \in L(s)$ iff $N, s \models \sim p$. (2): $M, s \models^{-} \sim p$ iff $M, s \models^{+} p$ iff $p \in L^{+}(s)$ iff $p \in L(s)$ iff $N, s \models p$ iff $N, s \models \sim \sim p$.

Induction step: We show only some cases.

(Case $\alpha \equiv \sim \beta$ ): (1): $M, s \models^{+} \sim \beta$ iff $M, s \models^{-} \beta$ iff $N, s \models \sim \beta$ (induction hypothesis). (2): $M, s \models^{-} \sim \beta$ iff $M, s \models^{+} \beta$ iff $N, s \models \beta$ (induction hypothesis) iff $N, s \models \sim \sim \beta$.

(Case $\left.\alpha \equiv \alpha_{1} \wedge \alpha_{2}\right):(1): M, s \models^{+} \alpha_{1} \wedge \alpha_{2}$ iff $M, s \models^{+} \alpha_{1}$ and $M, s \models^{+} \alpha_{2}$ iff $N, s \models \alpha_{1}$ and $N, s \models \alpha_{2}$ (induction hypothesis) iff $N, s \models \alpha_{1} \wedge \alpha_{2}$. (2): $M, s \models^{-} \alpha_{1} \wedge \alpha_{2}$ iff $M, s \models^{-} \alpha_{1}$ or $M, s \models^{-} \alpha_{2}$ iff $N, s \models \sim \alpha_{1}$ or $N, s \models \sim \alpha_{2}$ (induction hypothesis) iff $N, s \models \sim\left(\alpha_{1} \wedge \alpha_{2}\right)$.

(Case $\alpha \equiv \mathrm{A} \beta)$ : (1): $M, s \models^{+} \mathrm{A} \beta$ iff $\forall \pi$ : path starting from $s\left[M, \pi \models^{+}\right.$ $\beta]$ iff $\forall \pi$ : path starting from $s[N, \pi \models \beta]$ (induction hypothesis) iff $N, s \models$ $\mathrm{A} \beta$. (2): $M, s \models^{-} \mathrm{A} \beta$ iff $\exists \pi$ : path starting from $s\left[M, \pi \models^{-} \beta\right]$ iff $\exists \pi$ : path starting from $s[N, \pi \models \sim \beta]$ (induction hypothesis) iff $N, s \models \sim \mathrm{A} \beta$.

(Case $\alpha \equiv \beta_{1} \mathrm{U} \beta_{2}$ and $s$ is a path $\pi$ ): (1): $M, \pi \models^{+} \beta_{1} \mathrm{U} \beta_{2}$ iff $\exists k \geqslant 0$ $\left[\left(M, \pi^{k} \models^{+} \beta_{2}\right)\right.$ and $\forall j\left(0 \leqslant j<k\right.$ implies $\left.\left.M, \pi^{j} \models^{+} \beta_{1}\right)\right]$ iff $\exists k \geqslant 0$ $\left[\left(N, \pi^{k} \models \beta_{2}\right)\right.$ and $\forall j\left(0 \leqslant j<k\right.$ implies $\left.\left.N, \pi^{j} \models \beta_{1}\right)\right]$ (induction hypothesis) iff $N, \pi \models \beta_{1} \mathrm{U} \beta_{2}$. (2): $M, \pi \models^{-} \beta_{1} \mathrm{U} \beta_{2}$ iff $\forall j \geqslant 0\left[\forall i<j\right.$ not- $\left[M, \pi^{i} \models^{-} \beta_{1}\right]$ implies $\left.M, \pi^{j} \models^{-} \beta_{2}\right]$ iff $\forall j \geqslant 0\left[\forall i<j\right.$ not- $\left[N, \pi^{i} \models \sim \beta_{1}\right]$ implies $N, \pi^{j} \models$ $\left.\sim \beta_{2}\right]$ (induction hypothesis) iff $N, \pi \models \sim\left(\beta_{1} \mathrm{U} \beta_{2}\right)$. 
Next, the logic CTL* is defined.

Definition 3.14. Let AT be a nonempty subset of ATOM. A Kripke structure for $\mathrm{CTL}^{*}$ is a single-consequence Kripke structure $\left\langle S, S_{0}, R, L\right\rangle$, where $L$ is a function from $S$ to the power set of AT. The consequence relation $\models$ on a Kripke structure for CTL* is obtained from Definition 3.12 by deleting the conditions 18-36.

In order to distinguish the consequence relations, expressions $\models_{4 \mathrm{CTL}^{*}}$ and $\models_{\mathrm{CTL}^{*}}$ are used for $4 \mathrm{CTL}^{*}$ and $\mathrm{CTL}^{*}$, respectively.

In the following, a translation function from $4 \mathrm{CTL}^{*}$ into $\mathrm{CTL}^{*}$ is introdued. This is an extension of the translation function originally presented by Rautenberg [31].

DeFinition 3.15. Let AT be a nonempty subset of ATOM and $\mathrm{AT}^{\prime}:=$ $\left\{p^{\prime} \mid p \in \mathrm{AT}\right\}$. The language $\mathcal{L}^{\sim}$ (the set of formulas) of $4 \mathrm{CTL}^{*}$ is defined by using AT, $\sim, \neg, \rightarrow, \wedge, \vee, \mathrm{E}, \mathrm{A}$, path, X, F, G, U and R. The language $\mathcal{L}$ of $\mathrm{CTL}^{*}$ is obtained from $\mathcal{L}^{\sim}$ by adding $\mathrm{AT}^{\prime}$ and by deleting $\sim$.

A function $f$ from $\mathcal{L}^{\sim}$ to $\mathcal{L}$ is defined as follows.

$$
\begin{aligned}
f(p) & :=p \text { and } f(\sim p):=p^{\prime} \in \mathrm{AT}^{\prime}, \text { for any } p \in \mathrm{AT}, \\
f(\alpha \circ \beta) & :=f(\alpha) \circ f(\beta), \text { where } \circ \in\{\wedge, \vee, \rightarrow, \mathrm{U}, \mathrm{R}\}, \\
f(\sim \sim \alpha) & :=f(\alpha), \\
f(\sim(\circ \alpha)) & :=\circ f(\sim \alpha), \text { where } \circ \in\{\neg, \mathrm{X}\}, \\
f(\sim(\alpha \wedge \beta)) & :=f(\sim \alpha) \vee f(\sim \beta), \\
f(\sim(\alpha \vee \beta)) & :=f(\sim \alpha) \wedge f(\sim \beta), \\
f(\sim(\alpha \rightarrow \beta)) & :=f(\alpha) \wedge f(\sim \beta), \\
f(\sim(\mathrm{E} \alpha)) & :=\mathrm{A} f(\sim \alpha), \\
f(\sim(\mathrm{A} \alpha))) & :=\mathrm{E} f(\sim \alpha), \\
f(\sim(\mathrm{F} \alpha)) & :=\mathrm{G} f(\sim \alpha), \\
f(\sim(\mathrm{G} \alpha)) & :=\mathrm{F} f(\sim \alpha), \\
f(\sim(\alpha \mathrm{U} \beta)) & :=f(\sim \alpha) \mathrm{R} f(\sim \beta), \\
f(\sim(\alpha \mathrm{R} \beta)) & :=f(\sim \alpha) \mathrm{U} f(\sim \beta) .
\end{aligned}
$$

Theorem 3.16. Let AT be a nonempty subset of ATOM, $\mathrm{AT}^{\sim}:=\{\sim p \mid p \in$ $\mathrm{AT}\}$ and $\mathrm{AT}^{\prime}:=\left\{p^{\prime} \mid p \in \mathrm{AT}\right\}$. Let $\alpha$ be a $4 \mathrm{CTL}^{*}$ formula in $\mathcal{L}^{\sim}$, and $f$ be a function defined in Definition 3.15. Suppose that $M$ is a single-consequence Kripke structure $\left\langle S, S_{0}, R, L\right\rangle$ for $4 \mathrm{CTL}^{*}$ with $L: S \rightarrow 2^{\mathrm{AT}} \cup \mathrm{AT}^{\sim}$, and that 
$N$ is a Kripke structure $\left\langle S, S_{0}, R, L^{\prime}\right\rangle$ for $\mathrm{CTL}^{*}$ with $L^{\prime}: S \rightarrow 2^{\mathrm{AT}^{\mathrm{TAT}}{ }^{\prime}}$ such that $L^{\prime}$ is obtained from $L$ by replacing $\mathrm{AT}^{\sim}$ by $\mathrm{AT}^{\prime}$ satisfying that $[p \in L(s)$ iff $\left.p \in L^{\prime}(s)\right]$ and $\left[\sim p \in L(s)\right.$ iff $\left.p^{\prime} \in L^{\prime}(s)\right]$. Let $s$ be a state in $S$ or a path in $M$ and $N$.

$$
M, s \models_{4 \mathrm{CTL}^{*}} \alpha \quad \text { iff } \quad N, s \models_{\mathrm{CTL}^{*}} f(\alpha) .
$$

Proof. This theorem is proved by induction on the complexity of $\alpha$.

Base step:

(Case $\alpha \equiv p \in \mathrm{AT}): M, s \models_{4 \mathrm{CTL}^{*}} p$ iff $p \in L(s)$ iff $p \in L^{\prime}(s)$ iff $N, s \models_{\mathrm{CTL}^{*}}$ $p$ iff $N, s \models_{\mathrm{CTL}^{*}} f(p)$.

(Case $\left.\alpha \equiv \sim p \in \mathrm{AT}^{\sim}\right): M, s \models_{4 \mathrm{CTL}^{*}} \sim p$ iff $\sim p \in L(s)$ iff $p^{\prime} \in L^{\prime}(s)$ iff $N, s \models_{\mathrm{CTL}^{*}} p^{\prime} \in \mathrm{AT}^{\prime}$ iff $N, s \models \mathrm{CTL}^{*} f(\sim p)$.

Induction step: We show only some cases.

(Case $\left.\alpha \equiv \sim \sim \alpha_{1}\right): \quad M, s \models_{4 \mathrm{CTL}^{*}} \sim \sim \alpha_{1}$ iff $M, s \models_{4 \mathrm{CTL}^{*}} \alpha_{1}$ iff $N, s \models_{\mathrm{CTL}^{*}} f\left(\alpha_{1}\right)$ (induction hypothesis) iff $N, s \models_{\mathrm{CTL}^{*}} f\left(\sim \sim \alpha_{1}\right)$.

(Case $\left.\alpha \equiv \sim\left(\alpha_{1} \wedge \alpha_{2}\right)\right): M, s \models_{4 \mathrm{CTL}^{*}} \sim\left(\alpha_{1} \wedge \alpha_{2}\right)$ iff $M, s \models_{4 \mathrm{CTL}^{*}} \sim \alpha_{1}$ or $M, s \models_{4 \mathrm{CTL}^{*}} \sim \alpha_{2}$ iff $N, s \models_{\mathrm{CTL}^{*}} f\left(\sim \alpha_{1}\right)$ or $N, s \models_{\mathrm{CTL}^{*}} f\left(\sim \alpha_{2}\right)$ (induction hypothesis) iff $N, s \models_{\mathrm{CTL}^{*}} f\left(\sim \alpha_{1}\right) \vee f\left(\sim \alpha_{2}\right)$ iff $N, s \models_{\mathrm{CTL}^{*}} f\left(\sim\left(\alpha_{1} \wedge \alpha_{2}\right)\right)$.

(Case $\alpha \equiv \sim \mathrm{E} \beta): \quad M, s \models_{4 \mathrm{CTL}^{*}} \sim \mathrm{E} \beta$ iff $\forall \pi$ : path starting from $s$ $\left[M, \pi \models_{4 \mathrm{CTL}^{*}} \sim \beta\right]$ iff $\forall \pi$ : path starting from $s\left[N, \pi \models_{\mathrm{CTL}^{*}} f(\sim \beta)\right]$ (induction hypothesis) iff $N, s \models_{\mathrm{CTL}^{*}} \mathrm{~A} f(\sim \beta)$ iff $N, s \models_{\mathrm{CTL}^{*}} f(\sim \mathrm{E} \beta)$.

(Case $\alpha \equiv \sim\left(\beta_{1} \mathrm{R} \beta_{2}\right)$ and $s$ is a path $\left.\pi\right): M, \pi \models_{4 \mathrm{CTL}^{*}} \sim\left(\beta_{1} \mathrm{R} \beta_{2}\right)$ iff $\exists k \geqslant$ $0\left[\left(M, \pi^{k} \models_{4 \mathrm{CTL}^{*}} \sim \beta_{2}\right)\right.$ and $\forall j\left(0 \leqslant j<k\right.$ implies $\left.\left.M, \pi^{j} \models_{4 \mathrm{CTL}^{*}} \sim \beta_{1}\right)\right]$ iff $\exists k \geqslant 0\left[\left(N, \pi^{k} \models \mathrm{CTL}^{*} f\left(\sim \beta_{2}\right)\right)\right.$ and $\forall j\left(0 \leqslant j<k\right.$ implies $N, \pi^{j} \models \mathrm{CTL}^{*}$ $\left.\left.f\left(\sim \beta_{1}\right)\right)\right]$ (induction hypothesis) iff $N, \pi \models_{\mathrm{CTL}^{*}} f\left(\sim \beta_{1}\right) \mathrm{U} f\left(\sim \beta_{2}\right)$ iff $N, \pi \models \mathrm{CTL}^{*} f\left(\sim\left(\beta_{1} \mathrm{R} \beta_{2}\right)\right)$.

By using Theorem 3.16, the existing model checking algorithms for CTL* can be used for 4CTL*: Assuming $\mathrm{AT}^{\prime}=\left\{p^{\prime} \mid p \in \mathrm{AT} \subseteq \mathrm{ATOM}\right\}$, the translation function $f$ interpretes formulas with $\sim$.

\section{4LCTL* and bisimulation}

\section{1. $4 \mathrm{LCTL}^{*}$}

Definition 4.17. Let Loc be a finite nonempty set of locations, and $l \in$ Loc. State and path formulas of 4LCTL* are obtained from Definition 2.1 by adding the following rules. (1) if $\alpha$ is a state formula, then so is $[l] \alpha .(2)$ if $\beta$ is a path formula, then so is $[l] \beta$. State and path formulas of $4 \mathrm{LCTL}^{*}$ are $4 \mathrm{LCTL}^{*}$ formulas. 
Definition 4.18. A locative Kripke structure is a structure $\left\langle\right.$ Loc, $S, S_{0}, R$, $\left.L^{+}, L^{-}\right\rangle$such that $S, S_{0}, R$ are the same as those in Definition $2.2, L^{+}, L^{-}$ are functions from $S \times$ Loc to the power set of the nonempty AT ( $\subseteq$ ATOM), and Loc is a finite nonempty set of locations.

A path in a locative Kripke structure can be defined similarly in Definition 2.3, which is independent of Loc.

DeFinition 4.19. Let AT be a nonempty subset of ATOM. Let $\alpha_{1}$ and $\alpha_{2}$ be state formulas of $4 \mathrm{LCTL}^{*}$ and, $\beta_{1}$ and $\beta_{2}$ be path formulas of $4 \mathrm{LCTL}^{*}$. Consequence relations $\models^{+}$and $\models^{-}$on a locative Kripke structure $M=$ $\left\langle\right.$ Loc, $\left.S, S_{0}, R, L^{+}, L^{-}\right\rangle$is defined inductively as follows $(\pi$ represents a path constructed from $S, s$ represents a state in $S$, and $l \in$ Loc):

1. $M,(s, l) \models^{+} p$ iff $p \in L^{+}(s, l)$ for $p \in$ AT and $l \in$ Loc,

2. $M,(s, l) \models^{-} p$ iff $p \in L^{-}(s, l)$ for $p \in$ AT and $l \in$ Loc,

3. $M,(s, l) \models[k] \alpha_{1}$ iff $M,(s, k) \models \alpha_{1}$,

4. $M,(s, l) \models^{+} \sim \alpha_{1}$ iff $M,(s, l) \models^{-} \alpha_{1}$,

5. $M,(s, l) \models^{+} \neg \alpha_{1}$ iff $\operatorname{not}-\left[M,(s, l) \models^{+} \alpha_{1}\right]$,

6. $M,(s, l) \models^{+} \alpha_{1} \wedge \alpha_{2}$ iff $M,(s, l) \models^{+} \alpha_{1}$ and $M,(s, l) \models^{+} \alpha_{2}$,

7. $M,(s, l) \models^{+} \alpha_{1} \vee \alpha_{2}$ iff $M,(s, l) \models^{+} \alpha_{1}$ or $M,(s, l) \models^{+} \alpha_{2}$,

8. $M,(s, l) \models^{+} \alpha_{1} \rightarrow \alpha_{2}$ iff $M,(s, l) \models^{+} \alpha_{1}$ implies $M,(s, l) \models^{+} \alpha_{2}$,

9. $M,(s, l) \models^{+} \mathrm{E} \beta_{1}$ iff there exists a path $\pi$ from $s$ such that $M,(\pi, l) \models^{+} \beta_{1}$,

10. $M,(s, l) \models^{+} \mathrm{A} \beta_{1}$ iff for every path $\pi$ starting from $s, M,(\pi, l) \models^{+} \beta_{1}$,

11. $M,(\pi, l) \models^{+} \operatorname{path}\left(\alpha_{1}\right)$ iff $s$ is the first state of $\pi$ and $M,(s, l) \models^{+} \alpha_{1}$,

12. $M,(\pi, l) \models[k] \beta_{1}$ iff $M,(\pi, k) \models \beta_{1}$,

13. $M,(\pi, l) \models^{+} \sim \beta_{1}$ iff $M,(\pi, l) \models^{-} \beta_{1}$,

14. $M,(\pi, l) \models^{+} \neg \beta_{1}$ iff $\operatorname{not}-\left[M,(\pi, l) \models^{+} \beta_{1}\right]$,

15. $M,(\pi, l) \models^{+} \beta_{1} \wedge \beta_{2}$ iff $M,(\pi, l) \models^{+} \beta_{1}$ and $M,(\pi, l) \models^{+} \beta_{2}$,

16. $M,(\pi, l) \models^{+} \beta_{1} \vee \beta_{2}$ iff $M,(\pi, l) \models^{+} \beta_{1}$ or $M,(\pi, l) \models^{+} \beta_{2}$,

17. $M,(\pi, l) \models^{+} \beta_{1} \rightarrow \beta_{2}$ iff $M,(\pi, l) \models^{+} \beta_{1}$ implies $M,(\pi, l) \models^{+} \beta_{2}$,

18. $M,(\pi, l) \models^{+} \mathrm{X} \beta_{1}$ iff $M,\left(\pi^{1}, l\right) \models^{+} \beta_{1}$,

19. $M,(\pi, l) \models^{+} \mathrm{F} \beta_{1}$ iff $\exists k \geqslant 0\left[M,\left(\pi^{k}, l\right) \models^{+} \beta_{1}\right]$, 
20. $M,(\pi, l) \models^{+} \mathrm{G} \beta_{1}$ iff $\forall k \geqslant 0\left[M,\left(\pi^{k}, l\right) \models^{+} \beta_{1}\right]$,

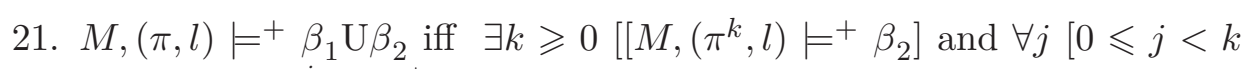
implies $\left.\left.M,\left(\pi^{j}, l\right) \models^{+} \beta_{1}\right]\right]$

22. $M,(\pi, l) \models^{+} \beta_{1} \mathrm{R} \beta_{2}$ iff $\forall j \geqslant 0\left[\forall i<j\right.$ not- $\left[M,\left(\pi^{i}, l\right) \models^{+} \beta_{1}\right]$ implies $\left.M,\left(\pi^{j}, l\right) \models+\beta_{2}\right]$

23. $M,(s, l) \models^{-} \sim \alpha_{1}$ iff $M,(s, l) \models^{+} \alpha_{1}$,

24. $M,(s, l) \models^{-} \neg \alpha_{1}$ iff $\operatorname{not}-\left[M,(s, l) \models^{-} \alpha_{1}\right]$,

25. $M,(s, l) \models^{-} \alpha_{1} \wedge \alpha_{2}$ iff $M,(s, l) \models^{-} \alpha_{1}$ or $M,(s, l) \models^{-} \alpha_{2}$,

26. $M,(s, l) \models^{-} \alpha_{1} \vee \alpha_{2}$ iff $M,(s, l) \models^{-} \alpha_{1}$ and $M,(s, l) \models^{-} \alpha_{2}$,

27. $M,(s, l) \models^{-} \alpha_{1} \rightarrow \alpha_{2}$ iff $M,(s, l) \models^{+} \alpha_{1}$ and $M,(s, l) \models^{-} \alpha_{2}$,

28. $M,(s, l) \models^{-} \mathrm{E} \beta_{1}$ iff for every path $\pi$ starting from $s, M,(\pi, l) \models^{-} \beta_{1}$,

29. $M,(s, l) \models^{-} \mathrm{A} \beta_{1}$ iff there exists a path $\pi$ from $s$ such that $M,(\pi, l) \models-\beta_{1}$,

30. $M,(\pi, l) \models^{-} \operatorname{path}\left(\alpha_{1}\right)$ iff $s$ is the first state of $\pi$ and $M,(s, l) \models^{-} \alpha_{1}$,

31. $M,(\pi, l) \models^{-} \sim \beta_{1}$ iff $M,(\pi, l) \models^{+} \beta_{1}$,

32. $M,(\pi, l) \models^{-} \neg \beta_{1}$ iff not- $\left[M,(\pi, l) \models^{-} \beta_{1}\right]$,

33. $M,(\pi, l) \models^{-} \beta_{1} \wedge \beta_{2}$ iff $M,(\pi, l) \models^{-} \beta_{1}$ or $M,(\pi, l) \models^{-} \beta_{2}$,

34. $M,(\pi, l) \models^{-} \beta_{1} \vee \beta_{2}$ iff $M,(\pi, l) \models^{-} \beta_{1}$ and $M,(\pi, l) \models^{-} \beta_{2}$,

35. $M,(\pi, l) \models^{-} \beta_{1} \rightarrow \beta_{2}$ iff $M,(\pi, l) \models^{+} \beta_{1}$ and $M,(\pi, l) \models^{-} \beta_{2}$,

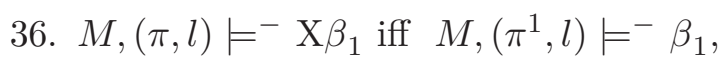

37. $M,(\pi, l) \models^{-} \mathrm{F} \beta_{1}$ iff $\forall k \geqslant 0\left[M,\left(\pi^{k}, l\right) \models^{-} \beta_{1}\right]$,

38. $M,(\pi, l) \models^{-} \mathrm{G} \beta_{1}$ iff $\exists k \geqslant 0\left[M,\left(\pi^{k}, l\right) \models^{-} \beta_{1}\right]$,

39. $M,(\pi, l) \models^{-} \beta_{1} \mathrm{U} \beta_{2}$ iff $\forall j \geqslant 0\left[\forall i<j\right.$ not- $\left[M,\left(\pi^{i}, l\right) \models^{-} \beta_{1}\right]$ implies $\left.M,\left(\pi^{j}, l\right) \models^{-} \beta_{2}\right]$,

40. $M,(\pi, l) \models^{-} \beta_{1} \mathrm{R} \beta_{2}$ iff $\exists k \geqslant 0\left[\left[M,\left(\pi^{k}, l\right) \models^{-} \beta_{2}\right]\right.$ and $\forall j[0 \leqslant j<k$ implies $\left.\left.M,\left(\pi^{j}, l\right) \models^{-} \beta_{1}\right]\right]$.

$M \models^{*} \alpha$ is defined by $\forall s \in S \forall l \in \operatorname{Loc}\left[M,(s, l) \models^{*} \alpha\right]$.

An expression $M \models^{*} \alpha$ can intuitively be interpreted as "If a proposition $\alpha$ can be verified (or refuted) at any time in the future for all spaces in a world $M$, then the proposition is the eternal truth (or falsehood) in the world" [22]. The proposed setting of the location operator [l] represents the 
discrete space interpretation in which a location is regarded as a point and is independent of other locations.

To introduce $[l]$ is to allow the following axiom schemes with respect to $[l]$ : for any $l, l_{i}, l_{j} \in \mathrm{Loc}$,

$$
\begin{aligned}
& {\left[l_{i}\right]\left[l_{j}\right] \alpha \leftrightarrow\left[l_{i}\right] \alpha,} \\
& {[l](\alpha \sharp \beta) \leftrightarrow([l] \alpha) \sharp([l] \beta), \quad \sharp \in\{\wedge, \vee, \rightarrow, \mathrm{U}, \mathrm{R}\},} \\
& {[l](\sharp \alpha) \leftrightarrow \sharp([l] \alpha), \quad \sharp \in\{\sim, \neg, \mathrm{E}, \mathrm{A}, \mathrm{X}, \mathrm{F}, \mathrm{G}\},}
\end{aligned}
$$

where an expression $\alpha \leftrightarrow \beta$ means $(\alpha \rightarrow \beta) \wedge(\beta \rightarrow \alpha)$. The first axiom scheme $\left[l_{i}\right]\left[l_{j}\right] \alpha \leftrightarrow\left[l_{i}\right] \alpha$ intuitively means that each location $l$ is the absolute address of locations, i.e. the location $l$ refers to the same location anywhere [25]. The second and third axiom schemes intuitively mean that the truth is time- and space-independent, i.e. "space" is almost independent of "time". As mentioned in $[23,22]$, the following inference rule called space induction rule is also true:

$$
\frac{(\forall l \in \operatorname{Loc})([l] \alpha)}{\alpha} .
$$

In this rule, for example, if $\left[l_{1}\right] \alpha,\left[l_{2}\right] \alpha$ and $\left[l_{3}\right] \alpha$ with Loc $=\left\{l_{1}, l_{2}, l_{3}\right\}$ hold, then $\alpha$ holds. It is also remarked that if Loc $=\{l\}$, then $[l]$ derives the modal logic S4-like axiom schemes:

$$
\begin{aligned}
& {[l](\alpha \rightarrow \beta) \rightarrow([l] \alpha \rightarrow[l] \beta),} \\
& {[l] \alpha \rightarrow[l][l] \alpha,} \\
& {[l] \alpha \rightarrow \alpha,}
\end{aligned}
$$

and hence $[l]$ is more expressive (or stronger) than the S4-type modal operator. Since the case that Loc is empty corresponds to the $4 \mathrm{CTL}^{*}$ case, $4 \mathrm{LCTL}^{*}$ is regarded as a natural generalization or extension of both CTL* and $4 \mathrm{CTL}^{*}$.

\subsection{Bisimulation}

Definition 4.20. Let $M=\left\langle\operatorname{Loc}, S, S_{0}, R, L^{+}, L^{-}\right\rangle$and $M^{\prime}=\left\langle\right.$ Loc, $S^{\prime}, S_{0}^{\prime}$, $\left.R^{\prime}, L^{\prime+}, L^{\prime-}\right\rangle$ be locative Kripke structures with the common (nonempty) sets AT $\left(\subseteq\right.$ ATOM) and Loc. The definition of bisimulation w.r.t. $M$ and $M^{\prime}$ is almost the same as that in Definition 2.5, since the space domain Loc is independent of this definition. ${ }^{5}$ The notion of the 'corresponding paths' in locative Kripke structures is the same as that in Definition 2.6.

\footnotetext{
${ }^{5}$ Of course, the conditions $L^{+}(s)=L^{\prime+}\left(s^{\prime}\right)$ and $L^{-}(s)=L^{\prime-}\left(s^{\prime}\right)$ in Definition 2.5 must be replaced by $L^{+}(s, l)=L^{\prime+}\left(s^{\prime}, l\right)$ and $L^{-}(s, l)=L^{\prime-}\left(s^{\prime}, l\right)$, respectively.
} 
The lemma concerning the notion of the 'corresponding paths' also holds for the same setting as Lemma 2.7.

Lemma 4.21. Let $M$ and $M^{\prime}$ be locative Kripke structures with the common (nonempty) sets AT ( $\subseteq$ ATOM) and Loc, and $B$ be a bisimulation relation between $M$ and $M^{\prime}$. Let $\alpha$ be a 4LCTL* formula and $l \in$ Loc. Assume that $B\left(s, s^{\prime}\right)$ and that $\pi$ in $M$ and $\pi^{\prime}$ in $M^{\prime}$ are corresponding paths.

If $\alpha$ is a state formula, then

(1) $M,(s, l) \models^{+} \alpha$ iff $M^{\prime},\left(s^{\prime}, l\right) \models^{+} \alpha$,

(2) $M,(s, l) \models^{-} \alpha$ iff $M^{\prime},\left(s^{\prime}, l\right) \models^{-} \alpha$,

and if $\alpha$ is a path formula, then

(1) $M,(\pi, l) \models^{+} \alpha$ iff $M^{\prime},\left(\pi^{\prime}, l\right) \models^{+} \alpha$,

(2) $M,(\pi, l) \models^{-} \alpha$ iff $M^{\prime},\left(\pi^{\prime}, l\right) \models^{-} \alpha$.

Proof. This lemma is proved by induction on the complexity of $\alpha$. The proof is almost the same as that in Lemma 2.8. In the following, the locative Kripke structures $M$ and $M^{\prime}$ are omitted in the expressions. The base step is obvious. We only consider the following cases for the induction step.

(Case $\alpha \equiv[k] \alpha_{1}$ is a state formula or a path formula): $(s, l) \models^{*}[k] \alpha_{1}$ iff $(s, k) \models^{*} \alpha_{1}$ iff $\left(s^{\prime}, k\right) \models^{*} \alpha_{1}$ (induction hypothesis) iff $\left(s^{\prime}, l\right) \models^{*}[k] \alpha_{1}$.

(Case $\alpha \equiv \alpha_{1} \mathrm{U} \alpha_{2}$, a path formula): We only show: $(\pi, l) \models{ }^{*} \alpha$ implies $\left(\pi^{\prime}, l\right) \models^{*} \alpha$. The converse direction can be shown in a similar way. We only consider the case for $*=+$. Suppose $(\pi, l) \models^{+} \alpha_{1} \mathrm{U} \alpha_{2}$. Then we have $\exists k \geqslant 0$ $\left[\left[\left(\pi^{k}, l\right) \models^{+} \alpha_{2}\right]\right.$ and $\forall j\left[0 \leqslant j<k\right.$ implies $\left.\left.\left(\pi^{j}, l\right) \models^{+} \alpha_{1}\right]\right]$. Since by the assumption, $\pi$ and $\pi^{\prime}$ are corresponding paths, $\pi^{j}$ and $\pi^{\prime j}$ are corresponding paths for any $j$. Thus, by the induction hypothesis, we obtain $\left(\pi^{\prime k}, l\right) \models^{+} \alpha_{2}$ and $\forall j\left[0 \leqslant j<k\right.$ implies $\left.\left(\pi^{\prime j}, l\right) \models^{+} \alpha_{1}\right]$. Therefore $\left(\pi^{\prime}, l\right) \models^{+} \alpha_{1} \mathrm{U} \alpha_{2}$.

Theorem 4.22. Let $M$ and $M^{\prime}$ be locative Kripke structures and $B$ be a bisimulation relation between $M$ and $M^{\prime}$. If $B\left(s, s^{\prime}\right)$, then for every $4 \mathrm{LCTL}^{*}$ formula $\alpha$ and any $l \in$ Loc,

(1) $M,(s, l) \models{ }^{+} \alpha$ iff $M^{\prime},\left(s^{\prime}, l\right) \models^{+} \alpha$,

(2) $M,(s, l) \models{ }^{-} \alpha$ iff $M^{\prime},\left(s^{\prime}, l\right) \models{ }^{-} \alpha$.

Theorem 4.23. Suppose that locative Kripke structures $M$ and $M^{\prime}$ are bisimulation equivalent. For every 4LCTL* formula $\alpha$,

(1) $M \models^{+} \alpha$ iff $M^{\prime} \models^{+} \alpha$,

(2) $M \models^{-} \alpha$ iff $M^{\prime} \models^{-} \alpha$. 


\section{Illustrative examples}

\subsection{Bipolar preferences}

It is known that preference modeling is a basic activity for any type of decision aiding process. Classical logic is used as a description language in traditional preference modeling. On the other hand, the classical logic is not always suitable to formalize real-life situations, since it is unable to handle inconsistent and uncertain information. A issue in preference modeling is to represent preference statements of the types 'I prefer $\alpha$ to $\beta$ ', which is called a positive preference, and 'I disprefer $\alpha$ to $\beta$ ', which is called a negative preference. ${ }^{6}$ It is remarked that the negative preference is not always the converse of the positive preference, since the notion 'preferences' in our real life is uncertain and inconsistent. Both positive and negative preferences, which are also called bipolar preferences, have recently been modelled based on some non-classical logics (see e.g. $[2,4]$ and the references therein).

In the framework based on $4 \mathrm{CTL}^{*}$, the bipolar preference statements 'I prefer $\alpha$ to $\beta$ ' and 'I disprefer $\alpha$ to $\beta$ ' can respectively be expressed in a Kripke structure as single-step transitions $s_{i} \rightarrow s_{i+1}$ with $\beta \in L^{+}\left(s_{i}\right)$ and $\alpha \in L^{+}\left(s_{i+1}\right)$, and $t_{j} \rightarrow t_{j+1}$ with $\beta \in L^{-}\left(t_{j}\right)$ and $\alpha \in L^{-}\left(t_{j+1}\right)$. A generalization of positive (or negative) preference expressions: $s_{1} \rightarrow s_{2} \rightarrow$ $s_{3} \rightarrow \cdots \rightarrow s_{n}$ where $L^{+}\left(s_{i}\right)=\alpha_{i}$ (or $L^{-}\left(s_{i}\right)=\alpha_{i}$ ) means an ascending order $\alpha_{1} \leqslant \alpha_{2} \leqslant \alpha_{3} \leqslant \cdots \leqslant \alpha_{n}$ of positive (or negative) preferences.

In the framework based on the located version $4 \mathrm{LCTL}^{*}$, information on locations can be added to the preference descriptions. For example, the statement 'Japanease people disprefer apple to orange in general' can be expressed as $s_{i} \rightarrow s_{i+1}$ with orange $\in L^{-}\left(s_{i}\right.$, Japan $)$ and apple $\in L^{-}\left(s_{i+1}\right.$, Japan $)$, where Loc $=\{$ Japan, China, $\ldots\}$.

\subsection{Taxonomic trees}

An example for taxonomic hierarchies with bipolar preferences for foods is addressed based on the following two taxonomic trees.

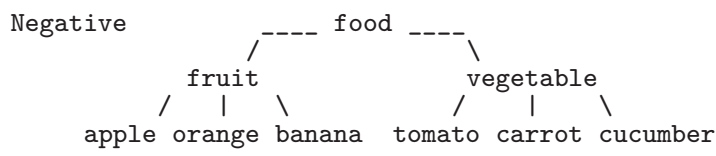

\footnotetext{
${ }^{6}$ It is explained in [4] that a positive preference is a statement of the type 'I like $\alpha$, and I like $\beta$ even more than $\alpha$ ', and a negative preference is a statement of the type 'I don't like $\alpha$, and I really don't like $\beta$ '.
} 


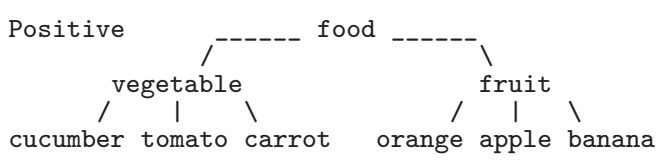

In these expressions, the negative preference orders are presented as

$$
\begin{aligned}
& \text { fruit } \leq \text { vegetable, } \\
& \text { apple } \leq \text { orange } \leq \text { banana } \leq \text { tomato } \leq \text { carrot } \leq \text { cucumber },
\end{aligned}
$$

and the positive preference orders are presented as

$$
\begin{aligned}
& \text { vegetable } \leq \text { fruit }, \\
& \text { cucumber } \leq \text { tomato } \leq \text { carrot } \leq \text { orange } \leq \text { apple } \leq \text { banana } .
\end{aligned}
$$

In order to represent the taxonomic trees using a Kripke structure, the following set of atomic formulas are assumed.

$$
\begin{aligned}
\mathrm{AT}= & \{\text { food }, \text { fruit }, \text { vegetable }, \text { apple }, \text { orange, banana }, \\
& \text { cucumber }, \text { tomato }, \text { carrot }\} .
\end{aligned}
$$

A Kripke structure $M=\left\langle S, S_{0}, R, L^{+}, L^{-}\right\rangle$for the taxonomic trees can be defined as

$$
\begin{aligned}
S & =\left\{s_{0}, s_{1}, s_{2}, s_{3}, s_{4}, s_{5}\right\}, \quad S_{0}=\left\{s_{0}\right\}, \\
R & =\left\{\left(s_{0}, s_{1}\right),\left(s_{1}, s_{2}\right),\left(s_{2}, s_{3}\right),\left(s_{3}, s_{4}\right),\left(s_{4}, s_{5}\right),\left(s_{5}, s_{5}\right)\right\}, \\
L^{-}\left(s_{0}\right) & =\{\text { food }, \text { fruit }, \text { apple }\} \\
L^{-}\left(s_{1}\right) & =\{\text { food, fruit, orange }\}, \\
L^{-}\left(s_{2}\right) & =\{\text { food, fruit, banana }\}, \\
L^{-}\left(s_{3}\right) & =\{\text { food, vegetable, tomato }\}, \\
L^{-}\left(s_{4}\right) & =\{\text { food, vegetable, carrot }\}, \\
L^{-}\left(s_{5}\right) & =\{\text { food, vegetable, cucumber }\}, \\
L^{+}\left(s_{0}\right) & =\{\text { food, vegetable, cucumber }\}, \\
L^{+}\left(s_{1}\right) & =\{\text { food, vegetable, tomato }\}, \\
L^{+}\left(s_{2}\right) & =\{\text { food, vegetable, carrot }\}, \\
L^{+}\left(s_{3}\right) & =\{\text { food, fruit, orange }\}, \\
L^{+}\left(s_{4}\right) & =\{\text { food, fruit, apple }\}, \\
L^{+}\left(s_{5}\right) & =\{\text { food, fruit, banana }\} .
\end{aligned}
$$


By using this structure, for example, the statement 'I prefer banana to apple' is expressed as $s_{4} \rightarrow s_{5}$ with apple $\in L\left(s_{4}\right)$ and banana $\in L\left(s_{5}\right)$, and the statement 'I disprefer vegetable to fruit' is expressed as $s_{2} \rightarrow s_{3}$ with fruit $\in L^{-}\left(s_{2}\right)$ and vegetable $\in L^{-}\left(s_{3}\right)$. This Kripke structure is regarded as a database representing the taxonomic trees for food preferences. Then we can check some properties for this database. For example, consider the following statements.

(1) There is 'orange' in this database, i.e. there is a state $s_{i}$ such that orange $\in L^{+}\left(s_{i}\right)$.

(2) 'Orange' is 'fruite', i.e. there is a state $s_{i}$ such that $\{$ orange, fruite $\} \subseteq$ $L^{+}\left(s_{i}\right)$.

(3) The root of the underlying taxonomic trees is 'food', i.e. the proposition food holds for all states.

These statements are respectively expressed as $M \models^{+}$AForange, $M \models^{+}$ $\mathrm{AF}$ (orange $\wedge$ fruit), and $M \models^{+}$AGfood.

It is remarked that by using the framework based on a locative Kripke structure for $4 \mathrm{LCTL}^{*}$, location information can be added to the expression defined above.

\subsection{Biomedical ontologies}

It is known that biomedical ontology constructions are important issues of life science, and various ontologies such as the Open Biomedical Ontologies $(\mathrm{OBO})$ and the Gene Ontologies (GO) have been proposed by many researchers (see e.g. [18, 32] and the references therein). Biomedical ontologies are a kind of knowledge representation models with hierarchies of biomedical vocabularies, called controlled vocabularies, and are usually represented as trees or directed acyclic graphs with some relations labelled by is-a (subtype relation), part-of (inclusion relation), located-in (spatial relation) and preceded-by (temporal relation) [32].

Tree and directed acyclic graph structures can appropriately be described by a (locative) Kripke structure, and the reasoning about time, space and uncertainty can naturally be represented by the virtue of $4 \mathrm{CTL}^{*}$ and $4 \mathrm{LCTL}^{*}$. A data base using such ontologies can thus be represented using a model checking framework based on the logics. A verification (or search) for a biological process called pathway can be performed by finding a path in a (locative) Kripke structure. 


\section{Concluding remarks}

In this paper, the bisimulation theorems for $4 \mathrm{CTL}^{*}$ and $4 \mathrm{LCTL}^{*}$ were shown, and the translation from $4 \mathrm{CTL}^{*}$ into $\mathrm{CTL}^{*}$ was presented using the singleconsequence Kripke structure for $4 \mathrm{CTL}^{*}$. In addition, some illustrative examples including bipolar preference modeling and taxonomic tree representation were presented based on $4 \mathrm{CTL}^{*}$ and $4 \mathrm{LCTL}^{*}$. It was thus shown that $4 \mathrm{CTL}^{*}$ and $4 \mathrm{LCTL}^{*}$ are useful as base logics for paraconsistent model checking: The bisimulation results guarantee to be able to apply abstraction, and the translation result allows us to use the existing CTL* model checking algorithms.

It is pointed out that although the idea of using two kinds of consequence relations $\models^{+}$and $\models^{-}$in Kripke structures is originally due to Routley [30], the idea of using a single consequence relation $\models$ with respect to $\sim$ is a new idea introduced in this paper, and the natural translation technique can thus be realized using the single-consequence Kripke structure. The single-consequence Kripke structure-based bisimulation theorem can also be shown for $4 \mathrm{CTL}^{*}$.

It is remarked that in a Kripke structure $\left\langle S, S_{0}, R, L^{+}, L^{-}\right\rangle$for $4 \mathrm{CTL}^{*}$, to adopt the condition $\forall s \in S\left[L^{+}(s) \cap L^{+}(s)=\emptyset\right]$ is equivalent to the fact that the underlying logic becomes a three-valued logic which is called here $3 \mathrm{CTL}^{*}$. Although $3 \mathrm{CTL}^{*}$ is not paraconsistent, the corresponding bisimulation and translation results can be obtained. These results for $3 \mathrm{CTL}^{*}$ can also be adapted for the locative version $3 \mathrm{LCTL}^{*}$. In addition, the bisimulation result with Loc can be adapted for the $\sim$-free part LCTL* of $3 \mathrm{LCTL}^{*}$ (or $4 \mathrm{LCTL}^{*}$ ), i.e. CTL* with the location operator.

\section{References}

[1] A. Almukdad and D. Nelson, "Constructible falsity and inexact predicates", Journal of Symbolic Logic 49 (1984), 231-233.

[2] S. Benferhat, D. Dubois, S. Kaci, and H. Prade, "Bipolar possibility theory in preference modeling: Representation, fusion and optimal solutions", Information Fusion 7(1) (2006), 135-150.

[3] J.-Y. Béziau, "The future of paraconsistent logic", Logical Studies 2 (1999). Online available. 
[4] S. Bistarelli, M.S. Pini, F. Rossi, and K.B. Venable, "Positive and negative preferences", Proceeding of the 7th International Workshop on Preferences and soft constraints (Soft 2005) held in conjunction with CP2005, Sitges, Spain, 2005.

[5] G. Bruns and P. Godefroid, "Model checking with multi-valued logics", Proceedings of ICALP2004, Lecture Notes in Computer Science 3142 (2004), pp. 281-293.

[6] M.C. Browne, E.M. Clarke, and O. Grumberg, "Characterizing finite Kripke structures in propositional temporal logic", Theoretical Computer Science 59 (1988), pp. 115-131.

[7] W.A. Carnielli, M.E. Coniglio, and J. Marcos, "Logics of formal inconsistency", in D. Gabbay and F. Guenthner (eds.), Handbook of Philosophical Logic, vol. 14, pp. 1-124, Kluwer Academic Publishers, 2004.

[8] M. Chechik, B. Devereux, S. Easterbrook, and A. Gurfinkel, "Multi-valued symbolic model-checking", ACM Transactions on Software Engineering and Methodology 12(4) (2004), 1-38.

[9] M. Chechik, and W. MacCaull, "CTL model-checking over logics with nonclassical negations", Proceedings of 33rd IEEE International Conference on Multi-valued logics (ISMVL'03), pp. 293-300, 2003.

[10] E.M. Clarke and E.A. Emerson, "Design and synthesis of synchronization skeletons using branching time temporal logic", Proceedings of the Workshop on logic of Programs, Lecture Notes in Computer Science 131 (1981), 52-71.

[11] E.M. Clarke, O. Grumberg, S. Jha, Y. Lu, and H. Veith, "Counterexampleguided abstraction refinement for symbolic model checking", Journal of the ACM 50(5) (2003), 752-794.

[12] E.M. Clarke, O. Grumberg, and D.A. Peled, Model checking, The MIT Press, 1999.

[13] C.V. Damásio and L.M. Pereira, "A survey of paraconsistent semantics for logic programs", in D. Gabbay and P. Smets (eds.), Handbook of Defeasible Reasoning and Uncertainty Management Systems, vol. 2, pp. 421-320, Kluwer Academic Publishers, 1998.

[14] S. Easterbrook, and M. Chechik, "A framework for multi-valued reasoning over inconsistent viewpoints", Proceedings of the 23rd International Conference on Software Engineering (ICSE 2001), pp. 411-420.

[15] S. Easterbrook, and M. Chechik, "Automated paraconsistent reasoning via model cheking", Proceedings of IJCAI Inconsistency Workshop, 8 pages, 2001.

[16] E.A. Emerson and J.Y. Halpern, "'Sometimes' and 'not never' revisited: on branching versus linear time temporal logic", Journal of the ACM 33(1) (1986), $151-178$. 
[17] E.A. Emerson and P. Sistla, "Deciding full branching time logic", Information and Control 61 (1984), 175-201.

[18] Ken I. Fukuda, "Biomedical ontologies and their applications" (in Japanese), Journal of the Japanese Society for Artificial Intelligence 22(1) (2007), 70-76.

[19] A. Gurfinkel, O. Wei, and M. Chechik, "Yasm: a software model-checker for verification and refutation", Proceedings of Computer Aided Verification (CAV'06), pp. 170-174, 2006.

[20] A. Gurfinkel, and M. Chechik, "Why wast a perfectly good abstraction?", Proceedings of TACAS'06, Lecture Notes in Computer Science 3920 (2006), $212-226$.

[21] N. Kamide, "Gentzen-type methods for bilattice negation", Studia Logica 80 (2005), 265-289.

[22] N. Kamide, "A spatial modal logic with a location interpretation", Mathematical Logic Quarterly 51(4) (2005), 331-341.

[23] N. Kamide, "Linear and affine logics with temporal, spatial and epistemic operators", Theoretical Computer Science 353(1-3) (2006), 165-207.

[24] N. Kamide, "Foundations of paraconsistent resolution", Fundamenta Informaticce 71(4) (2006), 419-441.

[25] N. Kobayashi, T. Shimizu, and A. Yonezawa, "Distributed concurrent linear logic programming", Theoretical Computer Science 227 (1999), 185-220.

[26] D. Nelson, "Constructible falsity", Journal of Symbolic Logic 14 (1949), 16-26.

[27] G. Priest, and R. Routly, "Introduction: paraconsistent logics", Studia Logica 43 (1982), 3-16.

[28] A. Pnueli, "The temporal logic of programs", Proceedings of the 18th IEEE Symposium on Foundations of Computer Science, pp. 46-57, 1977.

[29] J.P. Quielle and J. Sifakis, "Specification and verification of concurrent systems in CESAR", Proceedings of the 5th International Symposium on Programming, pp. 337-351, 1982 .

[30] R. Routley, "Semantical analysis of propositional systems of Fitch and Nelson", Studia Logica 33 (1974), 283-298.

[31] W. Rautenberg, Klassische und nicht-klassische Aussagenlogik, Vieweg, Braunschweig, 1979.

[32] B. Smith, W. Ceusters, B. Klagges, J. Köhler, A. Kumar, J. Lomax, C. Mungall, F. Neuhaus, A.L. Rector, and C. Rosse, "Relations in biomedical ontologies", Genome Biology 6 (2005), R46, 15 pages. 
[33] G. Wagner, "Logic programming with strong negation and inexact predicates", Journal of Logic and Computation 1(6) (1991), 835-859.

[34] H. Wansing, "The logic of information structures", Lecture Notes in Artificial Intelligence 681 (1993), 163 pages.

NORIHIRO KAMIDE

Research Center for Verification and Semantics

National Institute of Advanced Industrial Science and Technology

5th Floor, Mitsui Sumitomo Kaijo Bldg.

1-2-14 Shin-Senri Nishi, Toyonaka, Osaka, 560-0083 Japan

kamide@mtc.biglobe.ne.jp 